\title{
The endoplasmic reticulum stress inhibitor salubrinal inhibits the activation of autophagy and neuroprotection induced by brain ischemic preconditioning
}

\author{
Bo GAO\#, Xiang-yang ZHANG\#, Rong HAN, Tong-tong ZHANG, Cheng CHEN, Zheng-hong QIN, Rui SHENG* \\ Department of Pharmacology and Laboratory of Aging and Nervous Diseases, Soochow University School of Pharmaceutical Sci- \\ ence, Suzhou 215123, China
}

\begin{abstract}
Aim: To investigate whether endoplasmic reticulum (ER) stress participates in the neuroprotective effects of ischemic preconditioning (IPC)-induced neuroprotection and autophagy activation in rat brains.

Methods: The right middle cerebral artery in SD rats was occluded for 10 min to induce focal cerebral IPC, and was occluded permanently $24 \mathrm{~h}$ later to induce permanent focal ischemia (PFI). ER stress inhibitor salubrinal (SAL) was injected via intracerebral ventricle infusion $10 \mathrm{~min}$ before the onset of IPC. Infarct volume and motor behavior deficits were examined after the ischemic insult. The protein levels of LC3, p62, HSP70, glucose-regulated protein 78 (GRP 78), p-elF2 $\alpha$ and caspase-12 in the ipsilateral cortex were analyzed using immunoblotting. LC3 expression pattern in the sections of ipsilateral cortex was observed with immunofluorescence.

Results: Pretreatment with SAL (150 pmol) abolished the neuroprotective effects of IPC, as evidenced by the significant increases in mortality, infarct volume and motor deficits after PFI. At the molecular levels, pretreatment with SAL (150 pmol) significantly increased p-eIF2 $\alpha$ level, and decreased GRP78 level after PFI, suggesting that SAL effectively inhibited ER stress in the cortex. Furthermore, the pretreatment with SAL blocked the IPC-induced upregulation of LC3-II and downregulation of p62 in the cortex, thus inhibiting the activation of autophagy. Moreover,SAL blocked the upregulation of HSP70, but significantly increased the cleaved caspase-12 level, thus promoting ER stress-dependent apoptotic signaling in the cortex.

Conclusion: ER stress-induced autophagy might contribute to the neuroprotective effect of brain ischemic preconditioning.
\end{abstract}

Keywords: salubrinal; cerebral ischemia; ischemic preconditioning; endoplasmic reticulum stress; autophagy; glucose-regulated protein 78 (GRP78); p-elF2 $\alpha$; LC3; p62; HSP70; caspase-12

Acta Pharmacologica Sinica (2013) 34: 657-666; doi: 10.1038/aps.2013.34; published online 22 Apr 2013

\section{Introduction}

The application of a sub-threshold ischemic insult to an organ may activate certain cellular pathways that help to reduce the amount of damage caused by subsequent severe ischemic episodes. This phenomenon is known as ischemic preconditioning (IPC). Studying IPC may provide insight into endogenous protective mechanisms that could be exploited therapeutically ${ }^{[1-4]}$. The endoplasmic reticulum (ER) is an organelle in which secretory or membrane proteins are synthesized. Four main factors are known to cause ER stress:

\footnotetext{
\# These authors contributed equally to this work.

* To whom correspondence should be addressed.

E-mail sheng_rui@163.com

Received 2013-01-02 Accepted 2013-03-12
}

a) glucose/nutrient deficiencies, b) the inhibition of protein glycosylation, c) the disruption of disulfide bond formation and d) calcium depletion ${ }^{[5]}$. ER stress triggers an evolutionarily conserved signaling pathway involving RNA-regulated protein kinase-like ER kinase (PERK) and cell eukaryotic initiation factor 2 (eIF2a) kinase that phosphorylate the a subunit of eIF2, leading to the attenuation of protein synthesis. PERK, which has an endonuclease activity, translocates to the nucleus and induces the expression of several ER chaperones such as GRP78, which prevents protein-protein aggregation and helps to refold the proteins. However, excessive or prolonged ER stress may lead to ER-dependent apoptosis through the activation of CHOP (C/EBP homologous protein, growth arrest and DNA damage inducible gene 153, GADD153) and caspase- $12^{[6,7]}$. Evidence has shown that mild ER stress, 
characterized by the upregulation of GRP78, is involved in preconditioning ${ }^{[8-11]}$, but the pathway through which ER stress promotes the neuroprotective effects of preconditioning remains to be elucidated.

Autophagy is an evolutionarily conserved pathway that involves the sequestration and delivery of cytoplasmic materials to the lysosomes, where cellular constituents are degraded and recycled ${ }^{[12-15]}$. Autophagy is known to be activated during ischemic insult, but its contribution to neuronal death/survival is still being debated ${ }^{[16-18]}$. Recent reports also suggested an association between preconditioning and autophagy in both the heart and brain ${ }^{[19-22]}$. In an earlier study, we demonstrated that the neuroprotection induced by IPC is mediated by autophagy in a rat model of IPC, and the pharmacological induction of autophagy mimics the neuroprotection of IPC ${ }^{[23]}$. Furthermore, preconditioning-induced autophagy was able to inhibit excessive ER related-apoptosis in lethal ischemia, suggesting a correlation between ER stress and autophagy during preconditioning ${ }^{[2]}$. Furthermore, mounting evidence has shown that ER stress contributes to the activation of autophagy ${ }^{[25-28]}$. We thus speculate that ischemic preconditioning might induce mild ER stress to initiate the autophagic pathway. To test this hypothesis, we used pharmacological approaches to modulate ER stress in a rodent model of cerebral ischemic preconditioning and determine whether ER stress participates in IPC-elicited neuroprotection and autophagy activation.

\section{Materials and methods}

\section{Experimental protocol}

Male Sprague-Dawley rats weighing 280-300 g were purchased from the Center for Experimental Animals of Soochow University (certificate № 20020008, Grade II). The study was approved by the ethics committee of Soochow University. All animal procedures followed the NIH Guidelines for the Care and Use of Laboratory Animals. In this study, five sets of rats were used. The first set of 80 rats was randomly divided into eight treatment groups: permanent focal cerebral ischemia (PFI) $12 \mathrm{~h}$ and $24 \mathrm{~h}$, focal ischemic preconditioning (IPC) + PFI $12 \mathrm{~h}$ and $24 \mathrm{~h}$, salubrinal (SAL, L, $75 \mathrm{pmol})+\mathrm{IPC}+\mathrm{PFI} 12 \mathrm{~h}$ and $24 \mathrm{~h}$ and SAL $(\mathrm{H}, 150 \mathrm{pmol})+\mathrm{IPC}+\mathrm{PFI} 12 \mathrm{~h}$ and $24 \mathrm{~h}$. These rats were used for the evaluations of infarct volume, neurological deficits and mortality rates. The second batch of 60 rats was randomly divided into 6 treatment groups: sham-operated $6 \mathrm{~h}$ and $24 \mathrm{~h}, \mathrm{IPC}+\mathrm{PFI} 6 \mathrm{~h}$ and $24 \mathrm{~h}$ and SAL+IPC+PFI 6 $\mathrm{h}$ and $24 \mathrm{~h}$. These rats were used for ER stress-related protein immunoblotting experiments. The third batch of 60 rats was randomly divided into 6 treatment groups: sham-operated $6 \mathrm{~h}$ and $24 \mathrm{~h}$, IPC $6 \mathrm{~h}$ and $24 \mathrm{~h}$ and SAL+IPC $6 \mathrm{~h}$ and $24 \mathrm{~h}$. These rats were used for autophagy-related protein immunoblotting experiments. The fourth batch of 30 rats was randomly divided into 3 treatment groups: sham-operated $12 \mathrm{~h}$, IPC+PFI $12 \mathrm{~h}$ and SAL+IPC+PFI $12 \mathrm{~h}$. These rats were used for ER stress-related protein immunoblotting experiments. The last batch of 18 rats was randomly divided into 3 groups: shamoperated $6 \mathrm{~h}$, IPC $6 \mathrm{~h}$ and SAL+IPC $6 \mathrm{~h}$. These rats were used for the examination of LC3 immunofluorescence.

\section{Rat focal ischemic preconditioning model}

Focal ischemic preconditioning (IPC) and/or permanent focal cerebral ischemia (PFI) models were produced using the suture occlusion technique ${ }^{[29,30]}$. Briefly, rats were anesthetized with an intraperitoneal injection of $4 \%$ chloral hydrate (350 mg/ kg). A 30-mm length of monofilament nylon suture ( $\Phi 0.22-0.24 \mathrm{~mm})$, with its tip rounded by heating near a flame, was inserted from the right common carotid artery (CCA) to the internal carotid artery (ICA) through the stump of the external carotid artery (ECA) and then advanced to the Circle of Willis to occlude the origin of the right middle cerebral artery. Transient focal ischemia (10 min duration) was used to induce IPC. After IPC induction, the suture was gently withdrawn to permit reperfusion. Twenty-four hours later, the suture was reintroduced into the ICA through the stump of the ECA to introduce PFI (permanent middle cerebral artery occlusion, pMCAO). The SAL+IPC+PFI group underwent SAL pretreatment, transient focal ischemia of $10 \mathrm{~min}$ and subsequent pMCAO $12 \mathrm{~h}$ or $24 \mathrm{~h}$, whereas the IPC+PFI group underwent vehicle treatment and transient focal ischemia of 10 min with subsequent pMCAO surgery. The PFI group underwent vehicle treatment and pMCAO for 12 or $24 \mathrm{~h}$. LaserDoppler flowmetry (LDF, ML191 Laser Doppler Blood Flow Meter, AD instruments, Bella Vista NSW, Australia) was used to monitor the blockade and recovery of cerebral blood flow. Body temperature was closely monitored with a rectal probe and maintained in the range of $37.0 \pm 0.5^{\circ} \mathrm{C}$ with a heating pad (Institute of Biomedical Engineering, CAMS and BME-412A RAT REGULATOR) during and after surgery until recovery from anesthesia. About 20\%-30\% of rats died within $24 \mathrm{~h}$ after the onset of ischemia and were excluded from further analysis. Rats suffering from tremor and seizure (incidence of less than $5 \%)$ were also excluded from further analysis.

\section{Evaluation of infarct volume and motor deficits}

The neurological deficits in rats subjected to PFI were evaluated in a blinded manner at 12 or $24 \mathrm{~h}$ after ischemia using a previously described protocol with the following scoring system $^{[29]}: 0$ points, rats move normally; 1 point, rats cannot fully stretch their left front legs; 2 points, rats turn around in a circle; 3 points, rats fall down to the left side; 4 points, rats cannot move by themselves; 5 points, rats lose consciousness. After scoring, the rats were sacrificed, and their brains were dissected and sliced in a plastic module (Harvard Apparatus, 3-mm thickness) and stained with 4\% 2,3,5-triphenyltetrazolium chloride (TTC, Sinopharm Chemical Reagent Co Ltd, 30187713, Shanghai, China) for $30 \mathrm{~min}$ and then fixed with $4 \%$ paraformaldehyde. SigmaScan Pro 5 image analysis software was used to measure brain infarct. To account for the infarct volume expansion due to edematous change, the infarct volume was calculated with the following formula: infarct volume $=($ red area of contralateral side - red area of ipsilateral side) $/$ total area $\times 100 \%{ }^{[30]}$. 


\section{Immunoblotting}

At $6 \mathrm{~h}$ or $24 \mathrm{~h}$ after IPC and/or PFI with SAL pretreatment, the brain tissues from the ischemic cortex of the right middle cerebral artery territory and the corresponding area in shamoperated rats were homogenized in a lysis buffer $[10 \mathrm{mmol} / \mathrm{L}$ Tris- $\mathrm{HCl}, \mathrm{pH} 7.4,150 \mathrm{mmol} / \mathrm{L} \mathrm{NaCl}, 1 \%$ Triton-100, 0.1\% SDS, $5 \mathrm{mmol} / \mathrm{L}$ ethylenediaminetetraacetic acid (EDTA), 1 mmol/L phenylmethylsulfonyl fluoride (PMSF), $0.28 \mathrm{U} / \mathrm{mL}$ aprotinin, $50 \mu \mathrm{g} / \mathrm{mL}$ leupeptin, $1 \mathrm{mmol} / \mathrm{L}$ benzamidine, 7 $\mu \mathrm{g} / \mathrm{mL}$ pepstatin $\mathrm{A}]^{[32]}$, and proteins were extracted from the homogenate. The protein concentrations were determined (SmartSpec3000 Spectrophotometer, Bio-Rad, CA, USA) using a BCA kit (Pierce, 23227, Rockford, IL, USA). A $60-\mu \mathrm{g}$ aliquot of proteins from each sample was separated using 10\% SDSPAGE and subsequently transferred to a nitrocellulose membrane. Then, the membranes were incubated with specific antibodies against LC3 (1:1000; Abcam, ab62721, University of Cambridge, UK), p62 (1:1000; Enzo Life Science, Farmingdale, NY, USA), phospho-eIF2a (Ser51, 1:1000; Cell Signaling Technology, 9721, Beverley, MA, USA), GRP78 (1:1000; Stressgen Bioreagents, SPA-826, Victoria, Canada), HSP70 (1:200; Santa Cruz Biotechnology, sc-24, CA, USA) or caspase-12 (1:1000; Chemicon, AB3613, CA, USA) at $4{ }^{\circ} \mathrm{C}$ overnight and subsequently incubated with a horseradish peroxidase-conjugated secondary antibody (1:5000; Jackson ImmunoResearch, antirabbit, 711-035-152, anti-mouse, 715-035-150, West Grove, PA, USA) at room temperature for $1 \mathrm{~h}$. Immunoreactivity was detected using the Odyssey Two-Color Infrared Imaging System (LI-COR, Lincoln, NE, USA) in accordance with the manufacturer's instructions. The membranes were reprobed with $\beta$-actin (1:5000; Sigma) after stripping (TBST with $2 \%$ $\beta$-mercaptoethanol, $65^{\circ} \mathrm{C}, 1 \mathrm{~h}$ ). The protein expression was analyzed quantitatively with Sigma Scan Pro 5 and normalized to the loading control.

\section{Immunofluorescence}

Rats were anesthetized with $4 \%$ chloral hydrate and perfused with PBS followed by PBS containing 4\% paraformaldehyde. Perfusion-fixed brains were post-fixed in PBS containing $4 \%$ paraformaldehyde overnight. Coronal sections of $10-\mu \mathrm{m}$ thickness were cut with a cryostat, incubated with PBS containing $0.1 \%$ Triton $\mathrm{X}-100$ and $1 \%$ BSA for $1 \mathrm{~h}$, and then rinsed with PBS three times. Brain sections were then incubated with antibodies against LC3 (1:500; MBL, PD014) in a humidified container at $4^{\circ} \mathrm{C}$ for $48 \mathrm{~h}$. The sections were rinsed three times in PBS and sequentially incubated with CY3-conjugated anti-rabbit IgG (1:800) in a humidified container for $2 \mathrm{~h}$. The sections were then washed three times in PBS, incubated with 4,6-diamidino-2-phenylindole (DAPI, 1:10000) for $10 \mathrm{~min}$ and finally washed in PBS and sealed with a coverslip. The slides were analyzed under a laser confocal microscope (Nikon D-Eclipse C1, Japan).

\section{Treatment with ER stress inhibitor}

To determine the effects of pretreatment with the ER stress inhibitor salubrinal (SAL; Calbiochem, 324895) ${ }^{[33]}$ on the acti- vation of autophagy and neuronal injury after IPC+PFI, rats were treated with an intracerebral ventricle (icv) injection of 75 pmol SAL (in $1 \mu \mathrm{L}$ ) or 150 pmol SAL (in $2 \mu \mathrm{L}$ ) 10 min before the onset of IPC. SAL was dissolved in dimethylsulfoxide (DMSO) and further diluted with saline (final DMSO concentration $<0.2 \%$ ). Control rats received an icv injection of the same volume of vehicle.

\section{Statistical analysis}

For infarct volumes and immunoblotting, significant differences between groups were calculated by one-way ANOVA, and intergroup comparisons (post-hoc analysis) were carried out with the Newman-Keuls test. The mortality of animals in each group was compared with the chi-squared test, while neurological deficit scores were compared using non-parametric statistics (Kruskal-Wallis test), and Dunn's test was used for post-hoc analysis. $P<0.05$ was considered significant.

\section{Results}

\section{SAL suppressed the neuroprotection induced by IPC}

To determine the contribution of ER stress to the neuroprotection elicited by ischemic preconditioning, the effects of a single icv injection of the ER stress inhibitor SAL (75 or 150 pmol) administered 10 min before IPC+PFI were examined. In our preliminary study, we found that IPC+SAL (150 pmol) pretreatment did not cause visible infarction in the cortex and striatum. In addition, ischemic preconditioning (10 min tMCAO) did not induce infarction in the rat brain (data not shown). In rats subjected to PFI for $24 \mathrm{~h}$, extensive infarction was detected in the ipsilateral cerebral cortical and subcortical areas over a series of brain sections. The IPC treatment significantly reduced the infarct volume and neurological deficits following subsequent PFI, as shown in the vehicle+IPC+PFI 24 h group (Figure 1A, 1B, 1C, $P<0.01$ vs the PFI group). However, SAL had only mild effects on infarction volume and neurological deficits ( $P>0.05$ vs the vehicle+IPC + PFI group). This observation might be attributed to the higher mortality of rats in the SAL pretreatment groups (SAL+IPC+PFI) than in those without SAL pretreatment (Figure 1D, $P<0.05$ vs the vehicle+IPC+PFI group).

We thus further evaluated the effects of SAL on infarct volume at twelve hours after PFI. In rats subjected to IPC+PFI for only $12 \mathrm{~h}$, pretreatment with 150 pmol SAL (SAL+IPC+PFI $12 \mathrm{~h}$ ) significantly increased the infarct volume compared with the control group (vehicle+IPC+PFI 12 h), as shown in Figure $1 \mathrm{E}$ and $1 \mathrm{~F}(P<0.05)$. These rats were also examined and scored for motor deficits using a 5-point scale, as described in the Methods section. Rats that were pretreated with SAL showed more severe motor behavioral deficits than those without SAL treatment $(P<0.05$, Figure $1 G)$. All of these results indicate that SAL blocked the neuroprotection induced by ischemic preconditioning.

\section{SAL inhibited ER stress}

We examined the phosphorylation of eIF2a as an index of ER stress in the cortex after SAL treatment at different time 
A

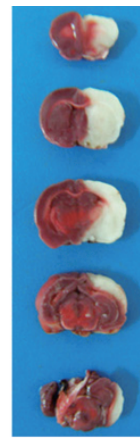

PFI 24 h

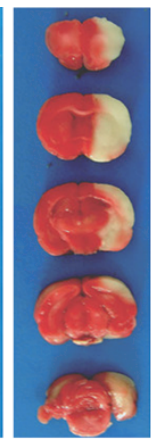

Vehicle+IPC PFI $24 \mathrm{~h}$
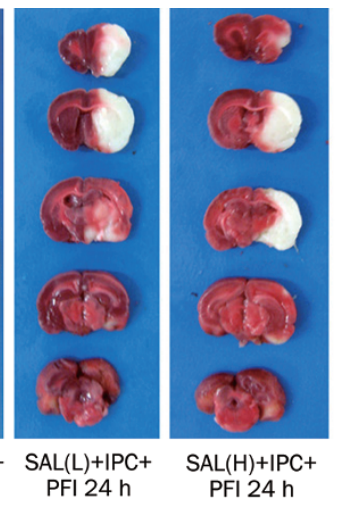

E

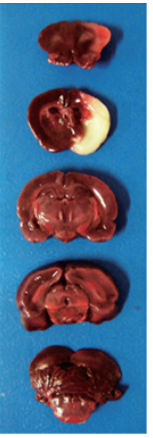

PFI $12 \mathrm{~h}$

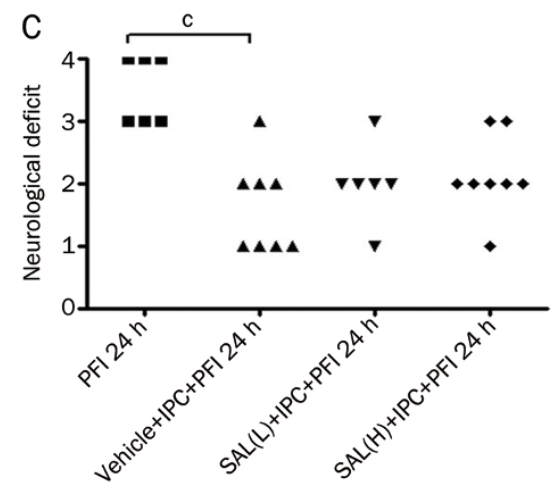

B

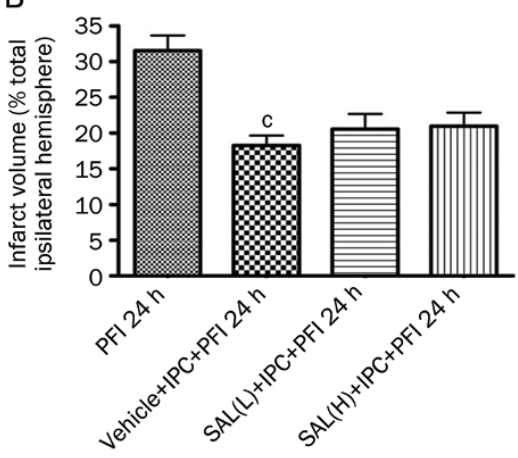

F

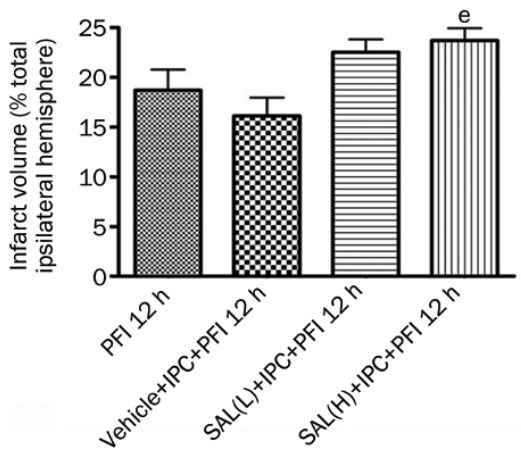

G

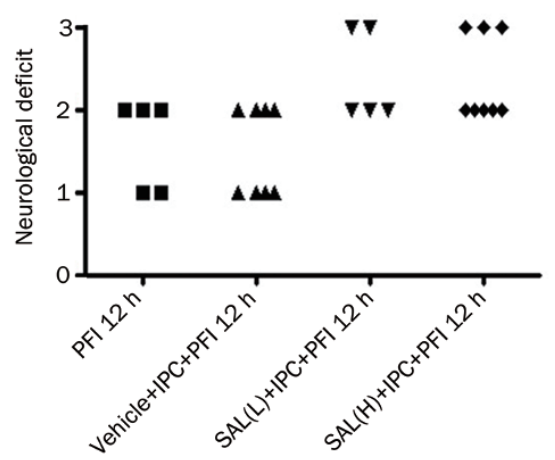

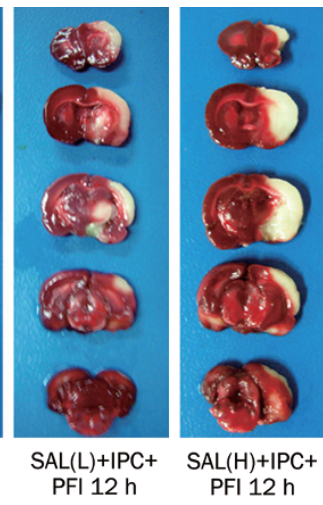

D

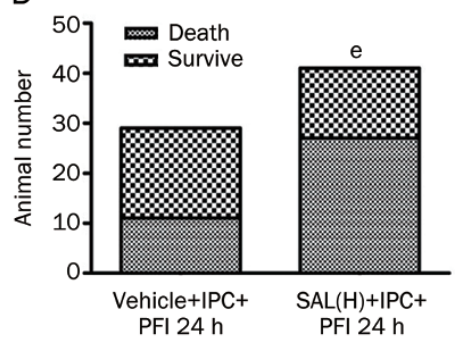

Figure 1. SAL pretreatment attenuated the neuroprotective effect of ischemic preconditioning (IPC) against subsequent lethal ischemia (PFI). Rats were treated with a single icv injection of SAL (75-150 pmol) 10 min before the onset of IPC (10 min MCAO). Lethal ischemia was induced by PFI (permanent focal cerebral ischemia). Rats were killed $12 \mathrm{~h}$ or $24 \mathrm{~h}$ after PFI. (A) TTC staining of brain sections showed the infarct volume at $24 \mathrm{~h}$ after PFI. (B) Quantitative analysis of brain infarct volume at $24 \mathrm{~h}$ after PFI. (C) Motor deficits at $24 \mathrm{~h}$ after PFI. (D) SAL treatment increased the mortality at $24 \mathrm{~h}$ after PFI. (E) TTC staining at $12 \mathrm{~h}$ after PFI. (F) Quantitative analysis of infarct volume at $12 \mathrm{~h}$ after PFI. (G) SAL treatment increased the motor deficits at $12 \mathrm{~h}$ after PFI. The bars represent the mean \pm SD. $n=5-8 .{ }^{\circ} P<0.01$ compared with the PFI group. ${ }^{\mathrm{e}} P<0.05$ compared with the IPC+PFI group. $\operatorname{SAL}(\mathrm{L})=\mathrm{SAL} 75 \mathrm{pmol} ; \mathrm{SAL}(\mathrm{H})=\mathrm{SAL} 150 \mathrm{pmol}$.

points. Western blot results revealed that SAL significantly inhibited the dephosphorylation of eIF2a in the cortex at $6 \mathrm{~h}$ after IPC+PFI (Figure 2A, $P<0.05$ vs the vehicle+IPC+PFI $6 \mathrm{~h}$ group). The protein levels of GRP78 were markedly reduced in the SAL-treated group compared with the vehicle+IPC+PFI $6 \mathrm{~h}$ group (Figure $2 \mathrm{~B}, P<0.05$ ). These results suggest that $\mathrm{SAL}$ treatment effectively inhibited ER stress in our experiments. Because SAL did not inhibit the dephosphorylation of eIF2a or GRP78 upregulation at $12 \mathrm{~h}$ or $24 \mathrm{~h}$ after IPC+PFI, we focused mainly on the effects of SAL on autophagy-related proteins and caspase-12 at the 6-h time point.

\section{SAL inhibited the IPC-induced activation of autophagy}

LC3 and p62 are two important biomarkers for autophagy activation in mammalian cells ${ }^{[35,36]}$. To examine the autophagic activity after SAL treatment, we examined the levels of the LC3 and p62 proteins in the cortex. The results showed that the level of the LC3-II protein $(16 \mathrm{kDa})$ was significantly 
A
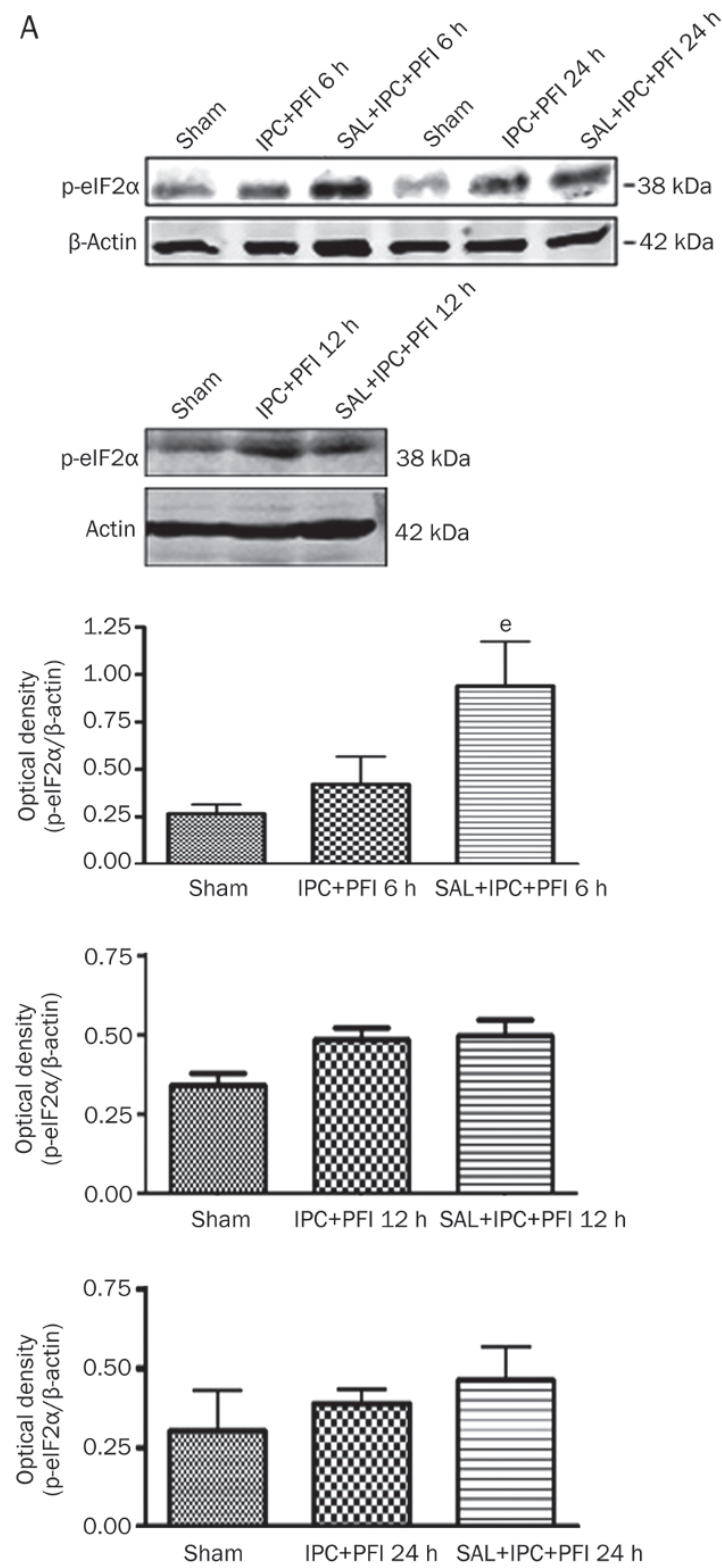

B
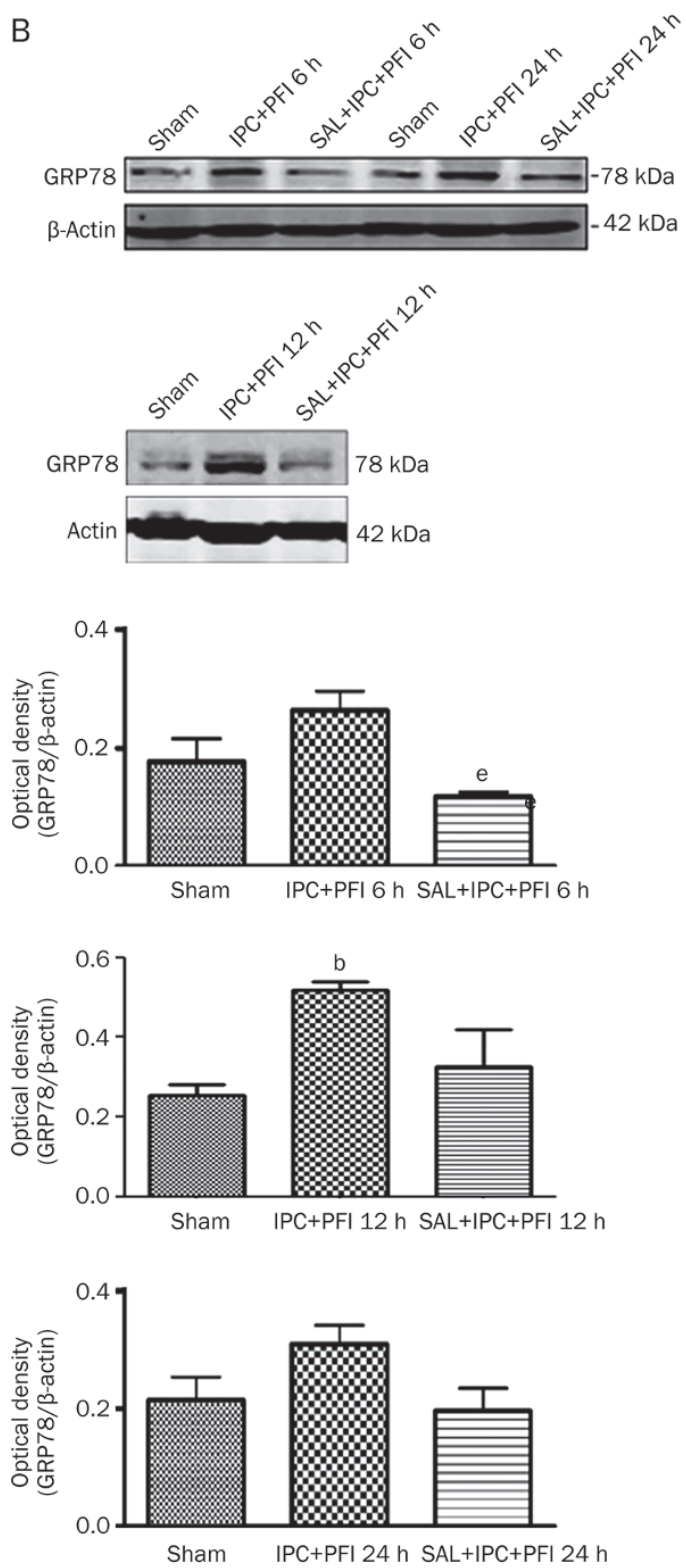

Figure 2. SAL inhibited the ER stress response in a rat model of ischemic preconditioning (IPC). Rats were treated with a single icv injection of $150 \mathrm{pmol}$ SAL $10 \mathrm{~min}$ before the onset of IPC. Lethal ischemia was induced by PFI. Rats were sacrificed at 6,12 , or $24 \mathrm{~h}$ after PFI. Extracts from ischemic and sham-operated cortex tissues were separated via SDS-PAGE, and protein levels were detected with immunoblotting. $\beta$-Actin was used as the loading control. Quantitative analysis was performed in Sigma Scan Pro 5. (A) Protein levels of p-EIF2 $\alpha$. (B) Protein levels of GRP78. The bars represent the mean \pm SD. $n=6$. ${ }^{b} P<0.05$ compared with the sham-operated group. ${ }^{e} P<0.05$ compared with the vehicle+IPC+PFI group.

upregulated in the cortex at $6 \mathrm{~h}$ after IPC $(P<0.05$ vs sham group, Figure 3A). However, the increase of LC3-II in the cortex was blunted by SAL treatment $(P<0.05$ vs IPC $6 \mathrm{~h}$ group). P62 expression was also significantly downregulated in the cortex at $6 \mathrm{~h}$ after IPC (Figure 3B; $P<0.05$ vs the shamoperated group), and the decrease of p62 expression in the cortex was significantly reversed by SAL $(P<0.01$ vs the IPC $6 \mathrm{~h}$ group).

To further evaluate the activation of autophagy, we examined LC3 in the cortex of the ipsilateral hemisphere via immunofluorescence. In the sham-operated group, the LC3 immu- noreactivity in cortex was low. Strong LC3 staining in cortical neurons was observed in the rats exposed to IPC and many LC3-positive neurons showed a punctate pattern (Figure 4). In contrast, in the SAL+IPC 6-h group, the LC3 immunoreactivity in these neurons was markedly lower. These results suggest that SAL significantly inhibited the autophagy activation induced by ischemic preconditioning.

\section{SAL promoted ER stress dependent apoptotic signaling}

Caspase-12 has been proposed as an important marker of ER stress-dependent apoptosis ${ }^{[34]}$, while HSP70 has neuroprotec- 
A
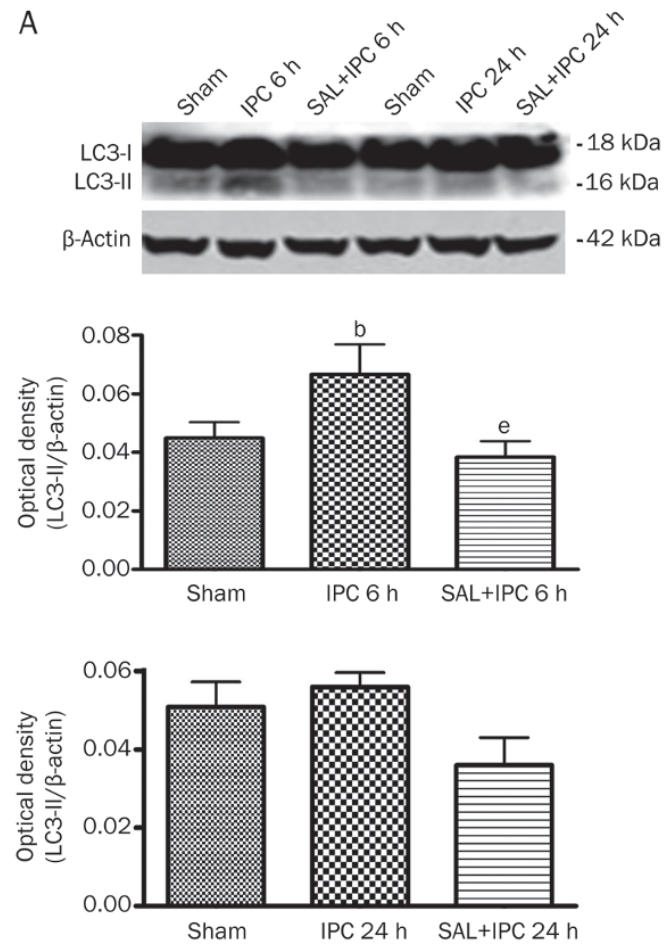

B
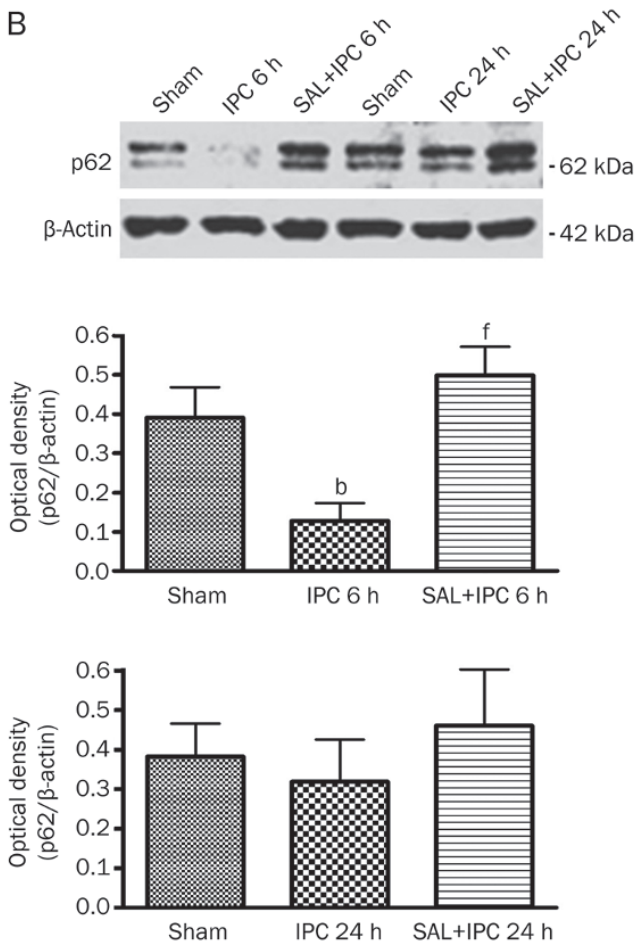

Figure 3. SAL inhibited the autophagy activation induced by ischemic preconditioning (IPC). Rats were treated with a single icv injection of 150 pmol SAL 10 min before the onset of IPC. Rats were sacrificed at 6 or $24 \mathrm{~h}$ after IPC. Protein extracts from ischemic and sham-operated cortex tissues were separated on SDS-PAGE, and the protein levels of LC3 and p62 were detected with immunoblotting. $\beta$-Actin was used as the loading control. Quantitative analysis was performed with Sigma Scan Pro 5. (A) Protein levels of LC3. (B) Protein levels of p62. The bars represent the mean \pm SD. $n=6 .{ }^{b} P<0.05$ compared with the sham-operated group. ${ }^{\mathrm{e}} P<0.05,{ }^{\mathrm{f}} P<0.01$ compared with the IPC group.

tive effects against apoptotic and necrotic cell death during cerebral ischemia. This study evaluated the effects of SAL on HSP70 and caspase-12. HSP70 protein expression was markedly lower in the SAL+IPC+PFI 6-h group than in the vehicle+IPC+PFI 6-h group (Figure $5 \mathrm{~A}, P<0.05$ ). In addition, the protein levels of cleaved caspase- 12 were significantly upregulated in both the SAL+IPC+PFI 6-h and 24-h groups compared with the corresponding vehicle+IPC + PFI groups (Figure $5 \mathrm{~B}, P<0.05$ ). These results suggest that although SAL inhibits ER stress, it might increase ER stress-dependent apoptotic signaling and reduce HSP70 expression; this might be one of the mechanisms by which SAL counteracts the neuroprotection mediated by IPC.

\section{Discussion}

This study was undertaken to explore whether ER stress is involved in the neuroprotective effects of IPC and participates in the pathways by which IPC activates autophagy. Our results demonstrated that the ER stress inhibitor SAL could partly abolish the neuroprotective effects of IPC, accompanied by a block of the ER stress and autophagy induced by preconditioning. These results strongly support the hypothesis that ER stress-induced autophagy is involved in IPC-elicited neuroprotection.

To determine the role of ER stress in the neuroprotection elicited by IPC, we investigated the effects of the ER stress inhibitor SAL in a rat model of focal ischemic preconditioning. In a previous study, we showed that SAL $75 \mathrm{pmol}$ $(\mathrm{SAL}+\mathrm{IPC}+\mathrm{PFI})$ has no significant effect on infarct volume compared with a control group (IPC+PFI) but significantly reduced the infarct volume and brain edema in the presence of 3-MA (SAL 75 pmol+3-MA+IPC+PFI), indicating that the inhibition of ER stress abolishes the brain damage induced by $3-\mathrm{MA}^{[24]}$. On the contrary, in this study, we found that SAL 150 pmol (SAL+IPC+PFI) pretreatment caused increased animal mortality $(24 \mathrm{~h})$, infarct volume $(12 \mathrm{~h})$ and neurological deficits $(12 \mathrm{~h})$ relative to those observed in the IPC+PFI group, suggesting that 150 pmol SAL suppresses the neuroprotective effects of preconditioning. Notably, we used SAL 75 pmol and $150 \mathrm{pmol}$ in the experiment, but SAL $75 \mathrm{pmol}$ had no significant effects, consistent with our previous findings ${ }^{[24]}$. SAL pretreatment increased the infarct volume and degree of motor deficits compared with IPC+PFI in the 12-h group but had no significant effects at $24 \mathrm{~h}$ after IPC+PFI. We think this discrepancy is mainly due to the high mortality. We noticed that most rats in the SAL $(150 \mathrm{pmol})+\mathrm{IPC}+\mathrm{PFI} 24 \mathrm{~h}$ group died overnight (12-24 h) after surgery. These rats, however, could not be used for TTC staining, affecting the infarct volume data.

SAL is the most commonly used ER stress inhibitor, and it acts by selectively inhibiting eIF2a dephosphorylation both in vitro and in vivo ${ }^{[33,37]}$. In this study, we first examined the expression of phosphorylated eIF2a in the cortex at $6 \mathrm{~h}, 12 \mathrm{~h}$ 

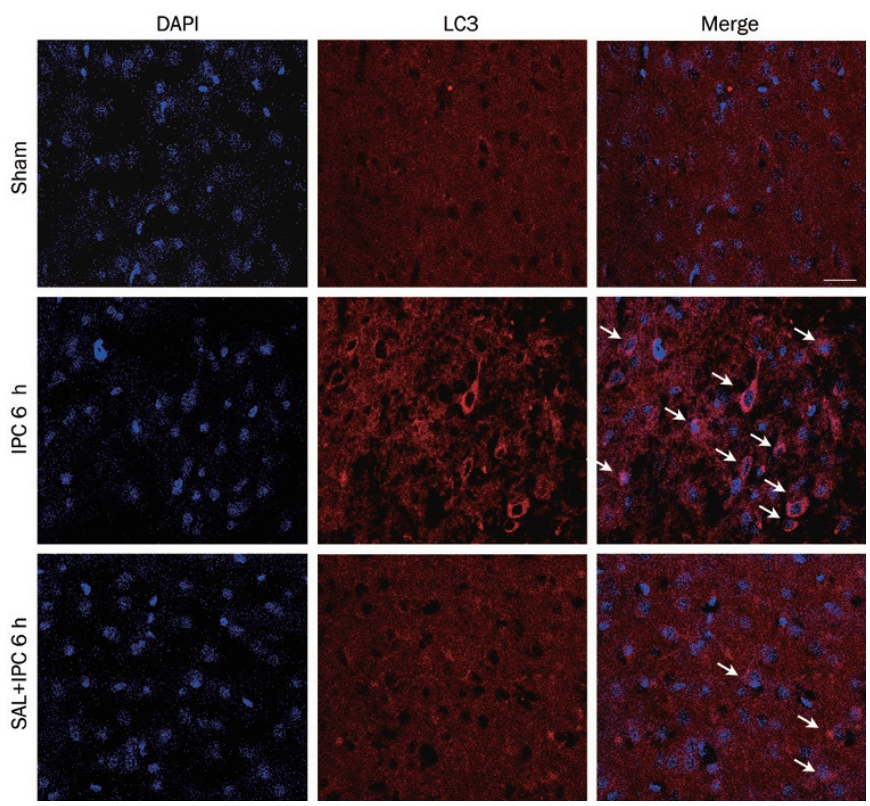

Figure 4. Cellular localization of LC3 immunofluorescence in the cortex after ischemic preconditioning (IPC). Sections of the ipsilateral hemisphere of the brain were double-labeled with anti-LC3 antibody (red) and DAPI (blue). Immunostained brain sections were analyzed with laser confocal microscopy. Note that LC3 expression was relatively low in the sham group. Strong LC3 staining in cortical neurons was observed in the rats exposed to IPC, and many LC3-positive neurons showed a punctate staining pattern. However, the LC3 immunoreactivity decreased markedly in the SAL+IPC 6 h group. $n=3$. Scale bar: $20 \mu \mathrm{m}$. Arrows indicate LC3positive neurons.

and $24 \mathrm{~h}$ after ischemic preconditioning. The results showed that SAL significantly inhibited the dephosphorylation of eIF2 $\alpha$ at $6 \mathrm{~h}$ after IPC+PFI, suggesting that SAL effectively inhibited ER stress in our experiments. During ER stress, cells first activate the unfolded protein response (UPR), which is characterized by the activation of three ER transmembrane effector proteins, PERK, IRE1, and ATF-6. One major pathway of the UPR involves the suppression of the majority of protein translation through the phosphorylation of eIF2a by PERK. Another pathway involves the upregulation of certain molecular chaperones, such as GRP78/Bip and GRP94 in the ER, which regulates protein folding and facilitates protein translocation and protein secretion in the $\mathrm{ER}^{[5-7]}$. It has been reported that SAL does not affect the UPR targets Xbp-1, GRP78, and GRP94 in PC12 cells treated for $36 \mathrm{~h}^{[33]}$. However, some studies have also reported that SAL could reduce the increase in GRP78 expression induced by a-TEA (RRR-a-tocopherol ether-linked acetic acid analog) or KA (kainic acid) ${ }^{[37,38]}$. This discrepancy is partly because previous research only examined the effects of SAL on normal cells. Consistent with the latter results, we found that SAL markedly reduced the protein levels of GRP78 at $6 \mathrm{~h}$ after IPC+PFI, further indicating that SAL effectively inhibited the early ER stress pathway in our experiment.

Several lines of evidence demonstrated that ER stress con- tributes to autophagy activation ${ }^{[25-28]}$. An essential step in ER stress-induced autophagosome formation is the phosphorylation of PERK/eIF2 $\alpha$. Once phosphorylated, eIF2 $\alpha$ can induce the production of LC3-II from LC3-I to induce autophagy ${ }^{[45]}$. We thus examined the protein levels of LC3 and p62 in the cortex to evaluate whether SAL could block the activation of autophagy by IPC. LC3, the microtubule-associated protein 1 A light chain 3, is a mammalian homologue of yeast ATG8. During the maturation of the autophagy response, LC3-I is converted to LC3-II by the cleavage of several amino acids at the C-terminus; therefore, autophagic activity, as measured by the number of autophagosomes, is directly correlated with LC3-II amount and the ratio of LC3-II (16 kDa) to LC3-I $(18 \mathrm{kDa})^{[35,46]}$. Another method for monitoring autophagy flow is to determine the levels of p62/SQSTM; p62 is associated with mature autophagic vesicles and is degraded within autophagosomes ${ }^{[36]}$. The results of immunoblotting and immunofluorescence assays showed that the LC3-II protein level was significantly upregulated while that of p62 was downregulated in the IPC 6-h group, and the changes in LC3-II and p62 expression in the ischemic cortex were significantly reversed by SAL pretreatment, indicating that SAL could block the autophagy activation induced by ischemic preconditioning.

The accumulation of unfolded proteins in the cytoplasm also induces the expression of molecular chaperones such as HSP70. HSP70 is the major stress-induced cytoplasmic chaperone, and its role in brain ischemia and ischemic tolerance has been studied intensively ${ }^{[39-42]}$. Recent research also implied that HSP70 might interact with the autophagy pathway to exert beneficial effects in neurodegenerative diseases ${ }^{[43,44]}$. In agreement with previous findings ${ }^{[8,40-42]}$, our results showed that the expression of HSP70 was upregulated after ischemic preconditioning. However, SAL treatment significantly inhibited the IPC-induced increases in HSP70, suggesting that SAL treatment effectively inhibits the survival pathway induced by ischemic preconditioning.

The initial response of ER stress and subsequent autophagy is to protect the cells by reestablishing homeostasis that is out of control due to ER stress, but ER stress is a double-edged sword; the degree of ER stress dictates to the cells whether to survive or die. If ER stress is mild, cells will express an adaptive response through autophagy and will survive during subsequent lethal ischemia. If ER stress is overwhelming, however, the cells will die through ER stress-related apoptosis $^{[26]}$. Caspase-12, a murine protein that is associated with the ER membrane, is involved in ER stress-dependent apoptosis. Caspase-12 normally exists in an inactive pro-caspase form ${ }^{[34]}$. During ER stress, caspase-12 dissociates from the ER membrane and is activated to initiate downstream apoptotic pathways. Caspase-12-deficient mice are resistant to ER stressdependent apoptosis. The present study demonstrated that pretreatment with SAL increased the activation of caspase-12, indicating that the suppression of the early ER stress protective response and autophagy pathway during preconditioning promote ER stress-dependent apoptosis.

One limitation of this study is that we did not examine the 
A
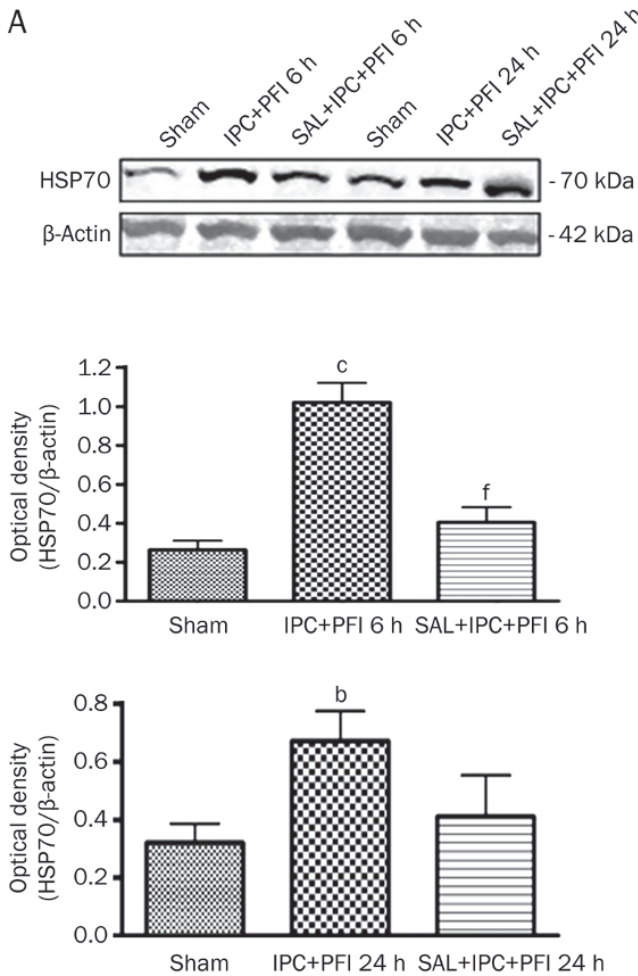

B
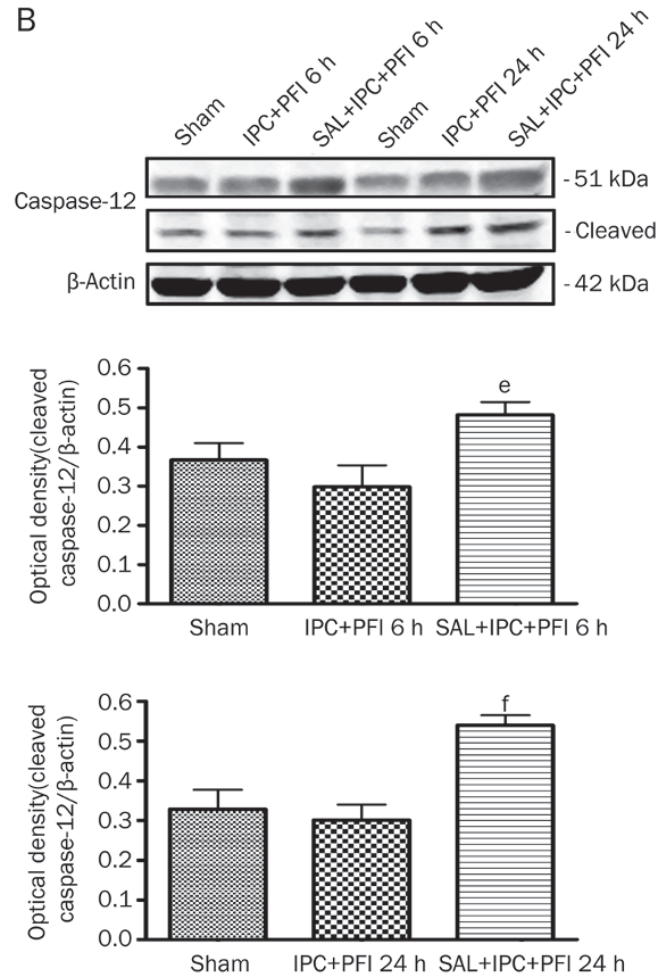

Figure 5. SAL promoted ER stress-dependent apoptosis in a rat model of ischemic preconditioning (IPC). Rats were treated with a single icv injection of $150 \mathrm{pmol}$ SAL $10 \mathrm{~min}$ before the onset of IPC. Lethal ischemia was induced by PFI. Rats were sacrificed at 6 or $24 \mathrm{~h}$ after PFI. Protein extracts from the ischemic and sham-operated cortex tissues were separated on SDS-PAGE, and the protein levels of caspase-12 and HSP70 were detected with immunoblotting. B-Actin protein was used as the loading control. Quantitative analysis was performed with Sigma Scan Pro 5 . (A) Protein levels of HSP70. (B) Protein levels of caspase-12. The bars represent the mean \pm SD. $n=6 .{ }^{b} P<0.05,{ }^{c} P<0.01$ compared with the sham-operated group. ${ }^{\mathrm{e}} P<0.05,{ }^{\mathrm{f}} P<0.01$ compared with the vehicle+IPC+PFI group..

biochemical alterations in the IPC or PFI alone groups because these experiments would have required an excessive number of rats and groups. However, our previous publications have shown that both IPC and PFI induced autophagy activation in the same IPC and PFI models, but the extent and persistence of autophagy activation varied ${ }^{[23]}$. IPC also upregulates HSP70 and GRP78 but downregulates cleaved caspase-12 relative to PFI $24 \mathrm{~h}$. Electron microscopy demonstrated the concurrence of mild ER stress and autophagy activation upon IPC treatment. However, the neurons in the PFI groups displayed much more severe ER stress and signs of cell injury. All of these results demonstrate that IPC elicits a low level of beneficial ER stress and autophagy to induce neuroprotection, while fatal ischemia induces prolonged and severe ER stress, leading to cell death ${ }^{[24]}$. Our present results showed that treatment with an ER stress inhibitor prevents ER stress and autophagy but upregulates caspase-12 expression, supporting the hypothesis that the inhibition of autophagy during preconditioning promotes caspase-12-dependent apoptosis in lethal ischemia. Based on the combination of the present results and our previous findings, we propose that preconditioning could induce mild ER stress, leading to autophagy, which prevents protein aggregation and excessive ER stress-induced apoptosis in subsequent lethal ischemia. Further investigation is needed to test this proposal.

In conclusion, our present results strongly suggest that ER stress-induced autophagy plays an important role in the neuroprotective effect of preconditioning. These findings may lead to novel strategies for the clinical prevention or treatment of ischemic stroke.

\section{Acknowledgements}

This work was supported by grants from National Natural Science Foundation of China (Grants 81173057, 30801391), and the Priority Academic Program of Jiangsu Higher Education Institutions (PAPD).

\section{Author contribution}

Rui SHENG and Zheng-hong QIN designed research; Bo GAO, Xiang-yang ZHANG, Tong-tong ZHANG, and Cheng CHEN performed research; Rong HAN contributed new analytical tools and reagents; Bo GAO analyzed data; Bo GAO, Rui SHENG, and Zheng-hong QIN wrote the paper.

\section{References}

1 Dirnagl U, Meisel A. Endogenous neuroprotection: mitochondria as gateways to cerebral preconditioning? Neuropharmacology 2008; 55: 334-44. 
2 Obrenovitch TP. Molecular physiology of preconditioning-induced brain tolerance to ischemia. Physiol Rev 2008; 88: 211-47.

3 Perez-Pinzon MA. Mechanisms of neuroprotection during ischemic preconditioning: Lessons from anoxic tolerance. Compara Biochem Physiol 2007; 147: 291-9.

4 Liu XQ, Sheng R, Qin ZH. The neuroprotective mechanism of brain ischemic preconditioning. Acta Pharmacol Sin 2009; 30: 1071-80.

5 Xu C, Bailly-Maitre B, Reed JC. Endoplasmic reticulum stress: cell life and death decisions. J Clin Invest 2005; 115: 2656-64.

6 Ma Y, Hendershot LM. The unfolding tale of the unfolded protein response. Cell 2001; 107: 827-30.

7 Boyce M, Yuan J. Cellular response to endoplasmic reticulum stress: a matter of life or death. Cell Death Differ 2006; 13: 363-73.

8 Hayashi T, Saito A, Okuno S, Ferrand-Drake M, Chan PH. Induction of GRP78 by ischemic preconditioning reduces endoplasmic reticulum stress and prevents delayed neuronal cell death. J Cereb Blood Flow Metab 2003; 23: 949-61.

9 Reddy RK, Mao C, Baumeister P, Austin RC, Kaufman RJ, Lee AS. Endoplasmic reticulum chaperone protein GRP78 protects cells from apoptosis induced by topoisomerase inhibitors: role of ATP binding site in suppression of caspase-7 activation. J Biol Chem 2003; 278: 20915-24.

10 Oidaa Y, Izutaa H, Oyagi A, Shimazawaa M, Kudob T, Imaizumic K, et al. Induction of $\mathrm{BiP}$, an ER-resident protein, prevents the neuronal death induced by transient forebrain ischemia in gerbil. Brain Res 2008; 1208: 217-24.

11 Yuan Y, Guo Q, Ye Z, Pingping X, Wang N, Song Z. Ischemic postconditioning protects brain from ischemia/reperfusion injury by attenuating endoplasmic reticulum stress-induced apoptosis through PI3K-Akt pathway. Brain Res 2011; 1367: 85-93.

12 Klionsky DJ, Emr SD. Autophagy as a regulated pathway of cellular degradation. Science 2000; 290: 1717-21.

13 Komatsu M, Waguri S, Chiba T, Murata S, Iwata J, Tanida I, et al. Loss of autophagy in the central nervous system causes neurodegeneration in mice. Nature 2006; 441: 880-4.

14 McCray BA, Taylor JP. The role of autophagy in age-related neurodegeneration. Neurosignals 2008; 16: 75-84.

15 Yen WL, Klionsky DJ. How to live long and prosper: autophagy, mitochondria, and aging. Physiology 2008; 23: 248-62.

16 Adhami F, Liao G, Morozov YM, Schloemer A, Schmithorst VJ, Lorenz $J N$. Cerebral ischemia-hypoxia induces intravascular coagulation and autophagy. Am J Pathol 2006; 169: 566-83.

17 Carloni S, Buonocore G, Balduini W. Protective role of autophagy in neonatal hypoxia-ischemia induced brain injury. Neurobiol Dis 2008; 32: 329-39.

18 Koike M, Shibata M, Tadakoshi M, Gotoh K, Komatsu M, Waguri S, et al. Inhibition of autophagy prevents hippocampal pyramidal neuron death after hypoxic-ischemic injury. Am J Pathol 2008; 172: 454-69.

19 Huang C, Yitzhaki S, Perry CN, Liu W, Giricz Z, Mentzer RM Jr, et al. Autophagy induced by ischemic preconditioning is essential for cardioprotection. J Cardiovasc Transl Res 2010; 3: 365-73.

20 Gurusamy N, Lekli I, Gorbunov NV, Gherghiceanu M, Popescu LM, Das DK. Cardioprotection by adaptation to ischaemia augments autophagy in association with BAG-1 protein. J Cell Mol Med 2009; 13: 373-87.

21 Yitzhaki S, Huang C, Liu W, Lee Y, Gustafsson AB, Mentzer RM Jr, et al. Autophagy is required for preconditioning by the adenosine $A 1$ receptor-selective agonist CCPA. Basic Res Cardiol 2009; 104: 15767.

22 Wu BX, Darden AG, Laser M, Li Y, Crosson CE, Hazard ES 3rd, et al. The rat Apg3p/Aut1p homolog is upregulated by ischemic precondi- tioning in the retina. Mol Vis 2006; 12: 1292-302.

23 Sheng R, Zhang LS, Han R, Liu XQ, Gao B, Qin ZH. Autophagy activation is associated with neuroprotection in a rat model of focal cerebral ischemic preconditioning. Autophagy 2010; 6: 482-94.

24 Sheng R, Liu XQ, Zhang LS, Gao B, Han R, Wu YQ, et al. Autophagy regulates endoplasmic reticulum stress in ischemic preconditioning. Autophagy 2012; 8: 310-25.

25 Yorimitsu T, Nair U, Yang Z, Klionsky DJ. Endoplasmic reticulum stress triggers autophagy. J Biol Chem 2006; 281: 30299-304.

26 Petrovski G, Das S, Juhasz B, Kertesz A, Tosaki A, Das DK. Cardioprotection by endoplasmic reticulum stress-induced autophagy. Antioxid Redox Signal 2011; 14: 2191-200.

27 Ogata M, Hino S, Saito A, Morikawa K, Kondo S, Kanemoto S, et al. Autophagy is activated for cell survival after endoplasmic reticulum stress. Mol Cell Biol 2006; 26: 9220-31.

28 Oh SH, Lim SC. Endoplasmic reticulum stress-mediated autophagy/ apoptosis induced by capsaicin (8-methyl-N-vanillyl-6-nonenamide) and dihydrocapsaicin is regulated by the extent of c-Jun $\mathrm{NH}_{2}$-terminal kinase/extracellular signal-regulated kinase activation in WI38 lung epithelial fibroblast cells. J Pharmacol Exp Ther 2009; 329: 112-22.

29 Longa EZ, Weinstein PR, Carlson S, Cummins R. Reversible middle cerebral artery occlusion without craniectomy in rats. Stroke 1989; 20: 84-91.

30 Lee SH, Kim YJ, Lee KM, Ryu S, Yoon BW. Ischemic preconditioning enhances neurogenesis in the subventricular zone. Neuroscience 2007; 146: 1020-31.

31 Tamura A, Graham DI, McCulloch J, Teasdale GM. Focal cerebral ischemia in the rat. 1 Description of technique and early neuropathological consequences following middle cerebral artery occlusion. J Cereb Blood Flow Metabol 1981; 1: 53-60.

32 Qin ZH, Chen RW, Wang Y, Nakai M, Chuang DM, Chase TN. Nuclear factor kappaB nuclear translocation upregulates c-Myc and p53 expression during NMDA receptor-mediated apoptosis in rat striatum. J Neurosci 1999; 19: 4023-33.

33 Boyce M, Bryant KF, Jousse C, Long K, Harding HP, Scheuner D, et al. A selective inhibitor of elF2 $\alpha$ dephosphorylation protects cells from ER stress. Science 2005; 307: 935-9.

34 Nakagawa, T, Zhu H, Morishima N, Li E, Xu J, Yankner BA, et al. Caspase-12 mediates endoplasmic-reticulum-specific apoptosis and cytotoxicity by amyloid-beta. Nature 2000; 403: 98-103.

35 Kabeya Y, Mizushima N, Ueno T, Yamamoto A, Kirisako T, Noda T, et al. LC3, a mammalian homologue of yeast Apg8p, is localized in autophagosome membranes after processing. EMBO J 2000; 19: 5720-8.

36 Chakrabarti L, Eng J, Ivanov N, Garden GA, La Spada AR. Autophagy activation and enhanced mitophagy characterize the Purkinje cells of pcd mice prior to neuronal death. Mol Brain 2009; 2: 24.

37 Sokka AL, Putkonen N, Mudo G, Pryazhnikov E, Reijonen S, Khiroug $\mathrm{L}$, et al. Endoplasmic reticulum stress inhibition protects against excitotoxic neuronal injury in the rat brain. J Neurosci 2007; 27: 901-8.

38 Tiwary R, Yu W, Li J, Park SK, Sanders BG, Kline K. Role of endoplasmic reticulum stress in alpha-TEA mediated TRAIL/DR5 death receptor dependent apoptosis. PLos One 2010; 5: e11865.

39 Chen J, Graham SH, Zhu RL, Simon RP. Stress proteins and tolerance to focal cerebral ischemia. J Cereb Blood Flow Metab 1996; 16: 566-77.

40 Currie RW, Ellison JA, White RF, Feuerstein GZ, Wang X, Barone FC. Benign focal ischemic preconditioning induces neuronal Hsp70 and prolonged astrogliosis with expression of Hsp27. Brain Res 2000; 863: 169-81.

41 Dhodda VK, Sailor KA, Bowen KK, Vemuganti R. Putative endogenous 
mediators of preconditioning-induced ischemic tolerance in rat brain identified by genomic and proteomic analysis. J Neurochem 2004; 89: 73-89.

42 Nishino K, Nowak TS Jr. Time course and cellular distribution of hsp27 and hsp72 stress protein expression in a quantitative gerbil model of ischemic injury and tolerance: thresholds for hsp72 induction and hilar lesioning in the context of ischemic preconditioning. J Cereb Blood Flow Metab 2004; 24: 167-78.

43 Massey AC, Kaushik S, Sovak G, Kiffin R, Cuervo AM. Consequences of the selective blockage of chaperone-mediated autophagy. Proc Natl Acad Sci U S A 2006; 103: 5805-10.

44 Meriin AB, Sherman MY. Role of molecular chaperones in neurodegenerative disorders. Int J Hyperthermia 2005; 21: 403-19.

45 Hoyer-Hansen M, Jaattela M. Connecting endoplasmic reticulum stress to autophagy by unfolded protein response and calcium. Cell Death Differ 2007; 14: 1576-82.

46 Kabeya Y, Mizushima N, Yamamoto A, Oshitani-Okamoto S, Ohsumi Y,
Yoshimori T. LC3, GABARAP and GATE16 localize to autophagosomal membrane depending on form-Il formation. J Cell Sci 2004; 117: 2805-12.

47 Kishi S, Shimoke K, Nakatani Y, Shimada T, Okumura N, Nagai K, et al. Nerve growth factor attenuates 2-deoxy-D-glucose-triggered endoplasmic reticulum stress-mediated apoptosis via enhanced expression of GRP78. Neurosci Res 2010; 66: 14-21.

48 Matus S, Lisbona F, Torres M, León C, Thielen P, Hetz C. The stress rheostat: an interplay between the unfolded protein response (UPR) and autophagy in neurodegeneration. Curr Mol Med 2008; 8: 15772.

49 Choi $\mathrm{CH}$, Jung $\mathrm{YK}$, Oh SH. Autophagy induction by capsaicin in malignant human breast cells is modulated by p38 and extracellular signal-regulated mitogen-activated protein kinases and retards cell death by suppressing endoplasmic reticulum stress-mediated apoptosis. Mol Pharmacol 2010; 78: 114-25. 